\title{
Buraco macular traumático bilateral pós-descarga elétrica. Considerações sobre um caso de etiologia incomum
}

\section{Macular hole following electric burn}

Nadyr Antonia Pereira Damasceno', Eduardo de França Damasceno², Soraya Alessandra Horowitz², Octavio Moura Brasil do Amaral Filho ${ }^{4}$

\section{RESUMO}

Os autores relatam um caso de buraco macular por descarga elétrica, enfatizando que sua fisiopatologia difere das causas comuns, como a traumática e idiopática. Descrevem alterações morfológicas características utilizando a tomografia de coerência óptica (OCT) e imagens de retinografia .

Descritores: Perfurações retinianas/etiologia; Acidentes por descargas elétricas/efeitos adversos; Tomografia de coerência óptica; Relatos de casos

'Médico do Setor de Retina e Vítreo do Hospital Naval Marcílio Dias - Rio de Janeiro (RJ), Brasil;

2Médico do Setor de Retina da Clínica de Olhos Octavio Moura Brasil - Rio de Janeiro (RJ), Brasil; Médico do Setor de Retina do Hospital Universitário Clemente Fraga Filho da Universidade Federal do Rio de Janeiro - UFRJ - Rio de Janeiro (RJ), Brasil;

${ }^{3}$ Médico do Setor de Retina e Vítreo do Hospital Naval Marcílio Dias - Rio de Janeiro (RJ), Brasil;

${ }^{4}$ Professor adjunto do Hospital Universitário Clementino Fraga Filho da Universidade Federal do Rio de Janeiro - UFRJ - Rio de Janeiro (RJ), Brasil.

Recebido para publicação em: 11/7/2008 - Aceito para publicação em 28/10/2008 


\section{INTRODUÇÃO}

A fisiopatologia do buraco macular traumático está correlacionado a lesões tipo "eyebal blow up" onde a súbita compressão e descompressão desencadeiam tração vitreoretiniana na mácula desencadeando o buraco por arrancamento ${ }^{(1-3)}$.

Entretanto, existe outra situação rara onde o trauma é o choque elétrico e a fisiopatogenia é a isquemia com nuances diferentes ${ }^{(4.8)}$.

Esta rara gênese é o objetivo deste trabalho.

\section{Relato de Caso}

Homem, negro, 57 anos, técnico eletricista, funcionário de subsidiária Light, apresentou-se com queixa de baixa acuidade visual bilateral de evolução há 5 semanas. Informou tratamento recente de ceratite epitelial com chamuscamento de cílios em ambos olhos, após exposição direta à descarga elétrica de transformador de alta voltagem (2000 volts).

Ao exame oftalmológico revelou: acuidade visual de 20/200 no olho direito e 20/400 no olho esquerdo; tonometria de aplanação de $15 \mathrm{mmHg}$ em ambos olhos (história de tratamento de glaucoma) e biomicroscopia demonstrando córnea com epitélio íntegro e sem evidência de pigmento na câmara anterior ou cristalino.

À biomicroscopia de fundo apresentou: Olho direito com buraco redondo em mácula (estágio III de Gass), papila com escavação de 0.5 e diminuição do calibre arteriolar (retinopatia hipertensiva); olho esquerdo com buraco macular total (estágio IV de Gass), papila com escavação de $0.8 \mathrm{H} / 0.7 \mathrm{~V}$ e diminuição do calibre arteriolar (retinopatia hipertensiva). Foi realizada, então, retinografia aneritra (Figuras 1 e2), além de tomografia de coerência óptica (OCT). Não foi feito exame de angiofluoresceinografia, porque o paciente referiu atopia ao contraste .

A imagem do OCT revelou uma formação de buraco macular completo no olho esquerdo, conforme a imagem da Figura 4, e no olho direito revelou buracomacular com espessamento e opérculo (Figura 3), pois há uma imagem de opérculo superior das camadas mais internas da retina, ainda com tração de hialóide posterior (classificação - estágio III de Gass ou II da classificação de OCT) ${ }^{(9-10)}$.

Conforme eleição do paciente, optou-se por uma conduta não invasiva e acompanhamento do quadro por curto a médio período de observação, não concordando com opção cirúrgica.

\section{Discussão}

O buraco macular com etiologia advinda de choque elétrico é desencadeado por ruptura das membranas celulares devido à geração de calor e vasoconstrição com

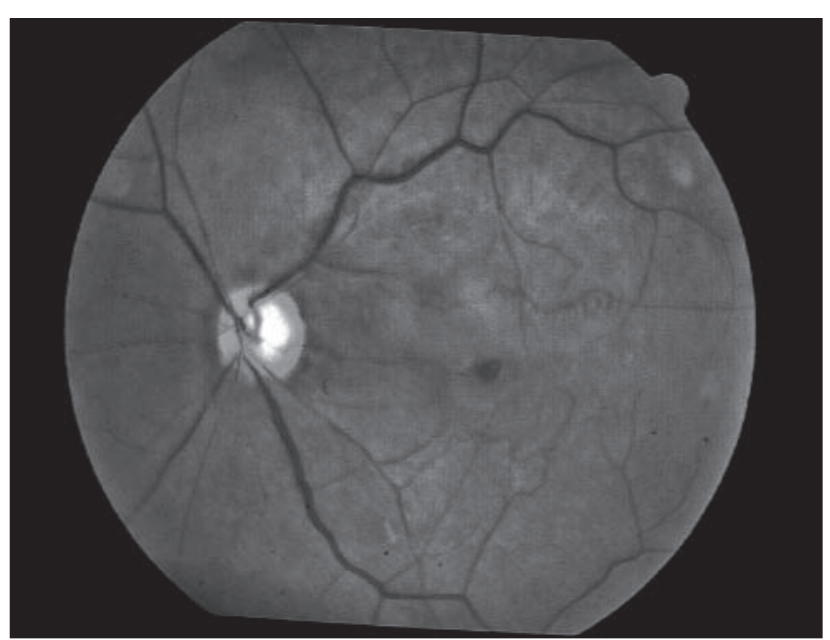

Figura 1: Retinografia aneritra - olho esquerdo - buraco macular

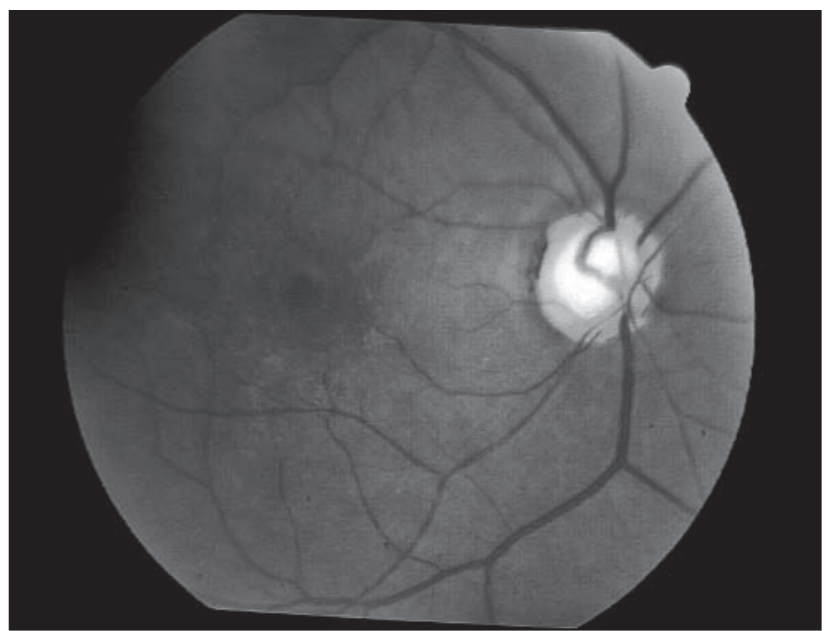

Figura 2: Retinografia aneritra - Olho direito - buraco macular aumento de escavação de disco óptico

conseqüente isquemia tecidual. A corrente elétrica estender-se-ia através do epitélio pigmentar da retina (EPR), em elevada concentração na mácula, e seria convertida em calor, destruindo a retina sobrejacente ${ }^{(6-7),(11-4)}$.

Além deste fato, outras injúrias oculares associadas seriam a quemose, ceratite punctata, lesão palpebral e catarata actínica ${ }^{(7)}$. Apesar de diferenças etiológicas entre o buraco macular idiopático, traumático e por choque elétrico, acredita-se que possa haver uma evolução similar à descrita por Gass 8, que descreve uma seqüência de apresentações evolutivas, iniciando com edema macular, formação de cisto e posteriormente buraco de mácula, o qual pode ter resolução espontânea ou não nestes períodos.

O OCT é um exame complementar de grande valia na distinção da entidade de buraco macular completo (de toda espessura), daqueles com acometimento parcial das camadas internas da retina, mes- 


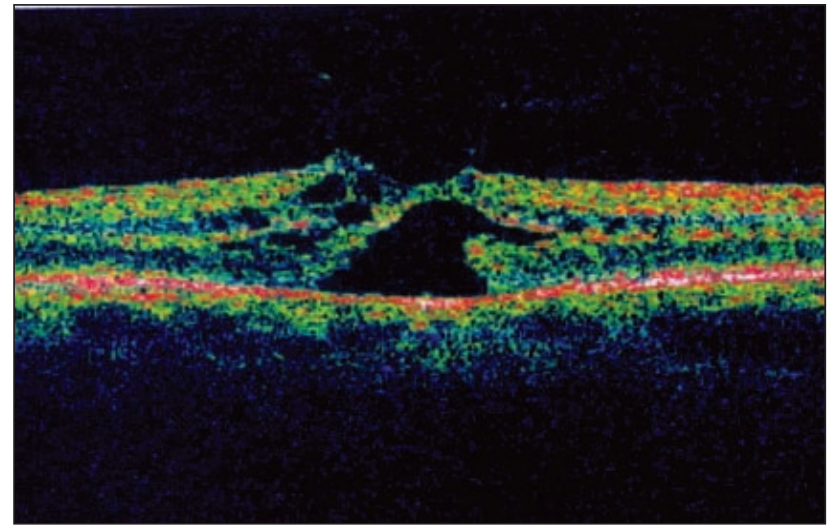

Figura 5: Olho Direito - OCT - Incidência de $90^{\circ}$ e $180^{\circ}$

mo com formações de pseudocistos e aumento de espessura macular ${ }^{(9-12)}$.

A possibilidade de uma indicação cirúrgica, com remoção da membrana limitante interna e hialóide posterior, foi a princípio aventada, devido à imagem da tração vítrea sobre o rebordo do buraco macular no olho direito, visto ao OCT. Entretanto, devido ao questionamento do seu efeito no resultado, sua fisiopatogenia e a recusa do paciente, não foi realizada ${ }^{(14-16)}$.

\section{Abstract}

The authors relate an uncommon case of macular hole following electric burn. They describe an uncommon physiopathology, which is different from traumatic and idiopathic macular hole.

Keywords: Retinal rerforations/etiology ; Accidents caused by electrical discharges/adverse effects; Tomography, optical coherence; Case reports

\section{REFERÊNCIAS}

1. Judson PH, Yanuzzi LA, Macular H. In: Ryan S. Retina. 3rd ed. St. Louis: Mosby; 2001.

2. Tavano V, Farah ME, Morales PHA, Mendaro AFN, Ferreira M, Bonomo PP. Epidemiologia do buraco macular . Arq Bras Oftalmol. 1998;61(1):77-81.

3. Bison SHDVF, Reggi JRA. Traumas oculares: nosologia de 1.171 casos. Arq Bras Oftalmol.1998;58(2:105-11.

4. Pieramici DJ, Sternberg P Jr, Aaberg TM Sr, Bridges WZ Jr, Capone A Jr, Cardillo JA, et al. A system for classifying mechanical injuries of the eye (globe). The Ocular Trauma Classification Group. Am J Ophthalmol. 1997;123(6):820-31.

5. Hirata A, Tanihara $\mathrm{H}$. uptured internal limiting membrane associated with blunt trauma revealed by indocyanine green staining. Graefes Arch Clin Exp Ophthalmol. 2004;242(6):527-30

6. Miller BK, Goldstein MH, Monshizadeh R, Tabandeh H, Bhatti MT. Ocular manifestations of electrical injury: a case report and review of the literature. CLAO J. 2002;28(4):224-7.

7. Al Rabiah SM, Archer DB, Millar R, Collins AD, Shepherd WF. Electrical injury of the eye. Int Ophthalmol. 1987;11(1):31-40
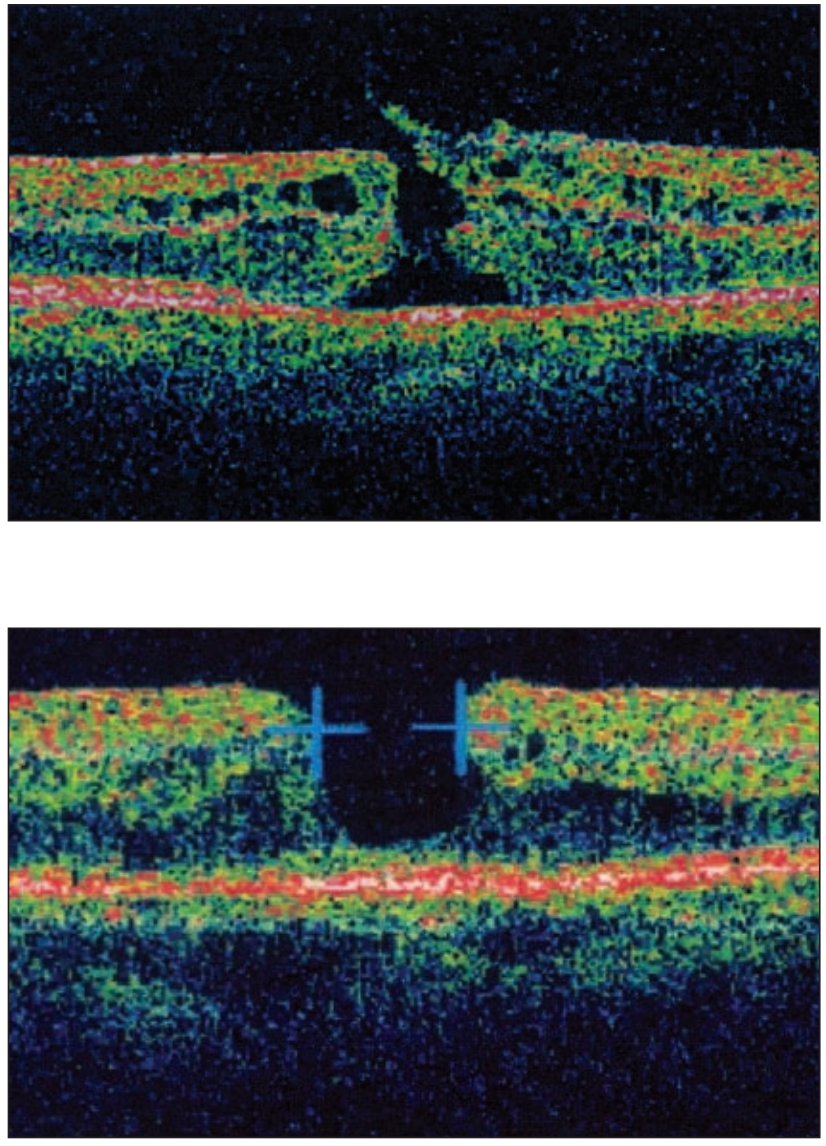

Figura 6: Olho Esquerdo - OCT - incidência de $90^{\circ}$

8. Gass JD. Reappraisal of biomicroscopic classification of stages of development of amacular hole. Am J Ophthalmol. $1995 ; 119(6): 752-9$.

9. Haouchine B, Massin P, Tadayoni R, Erginay A, Gaudric A. Diagnosis of macular pseudoholes and lamellar macular holes by optical coherence tomography. Am J Ophthalmol. 2004;138(5):732-9.

10. Hee MR, Puliafito CA, Wong C, Duker JS, Reichel E, Schuman JS, et al. Optical coherence tomography of macular holes. Ophthalmology. 1995;102(5):748-56.

11. Handa JT, Jaffe GJ. Lightning maculopathy. A case report. Retina. 1994;14(2):169-72.

12. Sony P, Venkatesh P, Tewari HK, Garg SP. Bilateral macular cysts following electric burn. Clin Experiment Ophthalmol. 2005;33(1):78-80

13. Manrique-Cerrillo M, Murillo-López S, Leizaola-Fernández C, Quiroz-Mercado H, Guerrero-Naranjo JL, Vargas-Castillo $\mathrm{R}$, et al. [Bilateral macular cysts secondary to electric current strike. A case report] Arch Soc Esp Oftalmol. 2004;79(1):379. Spanish.

14. Gardner TW, Ai E, Chrobak M, Shoch DE. Photic maculopathy secondary to short-circuiting of a high-tension electric current. Ophthalmology. 1982;89(7):865-8.

15. Johnson RN, McDonald HR, Lewis H, Grand MG, Murray TG, Mieler WF, et al. Traumatic macular hole: observations, pathogenesis, and results of vitrectomy surgery. Ophthalmology. 2001;108(5):853-7.

16. Spaide RF. Closure of an outer lamellar macular hole by vitrectomy: hypothesis for one mechanism of macular hole formation. Retina. 2000;20(6):587-90. 